\title{
Probing ancient mass loss with AKARl's extended thermal dust emission objects
}

\author{
Rachael L Tomasino ${ }^{1}$, Toshiya Ueta ${ }^{1,2}$, Yamamura Issei ${ }^{2}$, and Hideyuki Izumiura ${ }^{3}$ \\ ${ }^{1}$ University of Denver \\ ${ }^{2}$ Institute of Space and Astronautical Science-Japan Aerospace Exploration Agency \\ ${ }^{3}$ Okayama Astronomical Observatory \\ E-mail: Rachael.Tomasino@du.edu, Toshiya. Ueta@du.edu
}

\begin{abstract}
We present the method for the calibration and analysis of 166 far-IR extended thermal dust emission objects that were observed with AKARI's FIS detector. The primary goal of the analysis of this data set is to map the circumstellar shells of evolved stars in detail to understand the ancient history of dusty mass loss. After establishing an extended aperture photometry method, we re-characterize the flux dependent slow transient response correction factors for each of the four wavelength bands and propose a new flux calibration method for extended sources. Using these new correction factors, the photometric measurements are made using a extended aperture which is defined by a background $+3 \sigma$ threshold. With photometry measurements for each of the four FIS bands, an SED of a modified blackbody was fit to produce the emissivity beta, effective temperature, and color correction factor.
\end{abstract}

The Life Cycle of Dust in the Universe: Observations, Theory, and Laboratory Experiments - LCDU 2013, 18-22 November 2013

Taipei, Taiwan 


\section{Introduction}

Low to intermediate initial mass stars $\left(0.8-8 M_{\odot}\right)$ liberate much of their material through mass loss during the asymptotic giant branch phase. This liberation of the surface matter is thought to determine the course of the rest of the evolution to become a white dwarf. Unfortunately, our understanding of the evolution of the circumstellar shells remains qualitative at best, and therefore, observational characterization based on theoretical models is urgently needed.

Probing of the intrinsically cold (below $100 \mathrm{~K}$ ) circumstellar envelopes (CSEs) in the farinfrared (far-IR) began in the 1980s with the IRAS satellite [1] showing the existence of the CSEs as a consequence of continuous/continual dusty mass loss [2]. In the 1990s, observations made with ISO [3] confirmed that these CSEs were rich in molecules and dust [4] and their far-IR maps showed internal structures as signatures of mass-loss modulations [5]. Observations made at other wavelengths also indicated that variations in the rate of mass loss resulted in CSEs with multiple shells and axisymmetric structures [6]. In the early 21 st century, the new suite of far-IR satellites, Spitzer, AKARI, and Herschel, became available with higher spatial resolution and greater sensitivities $[7,8,9]$.

\section{Observations and data reduction}

AKARI is the first Japanese satellite dedicated to infrared astronomy [8]. The diameter of the telescope is $68.5 \mathrm{~cm}$ and is cooled down to $6 \mathrm{~K}$ in a liquid-helium cryostat for reducing the thermal emission. The Far-Infrared Surveyor (FIS) is one of the two focal-plane instruments on-board AKARI [10]. It has four photometric bands between 50-180 microns with two types of Ge:Ga array devices: the Short-Wavelength (SW) and the Long-Wavelength (LW) detectors.

Observational data for 165 extended emission objects and 27 reference point-source have been compiled to form the largest collection of one of the most sensitive far-IR images of the cold extended circumstellar dust shells of evolved stars. This data set is therefore the key to understanding the dusty mass loss phase of stellar evolution. The majority of data (143 objects) is from the MLHES (excavating Mass Loss History in Extended dust shells of Evolved Stars, PI: Yamamura) mission programme and others include data from the FISPN, WRENV, OBSTR, and DTFIS mission programmes $(7,10,5$, and 27 objects, respectively).

Due to the intrinsically faint nature of the extended far-IR cold dust shells, it is critical to have an optimized data reduction method to minimize the measurement uncertainties. This optimization was achieved with a new imaging tool kit FAST (FIS-AKARI Slow-scan Tools, [11]). FAST is a set of IDL programs that allows for interactive assessment of the data quality and on-the-fly corrections to the time-series data on a pixel-by-pixel basis. After applying FAST corrections, the nominal pipeline FIS Slow Scan tool was used to generate maps from the time-series scan-mapping data [12].

\section{Photometric flux calibration}

The Ge:Ga detectors used by FIS are known to show a slow transient response at low temperature under low background flux conditions, which typically happens in the space environment 

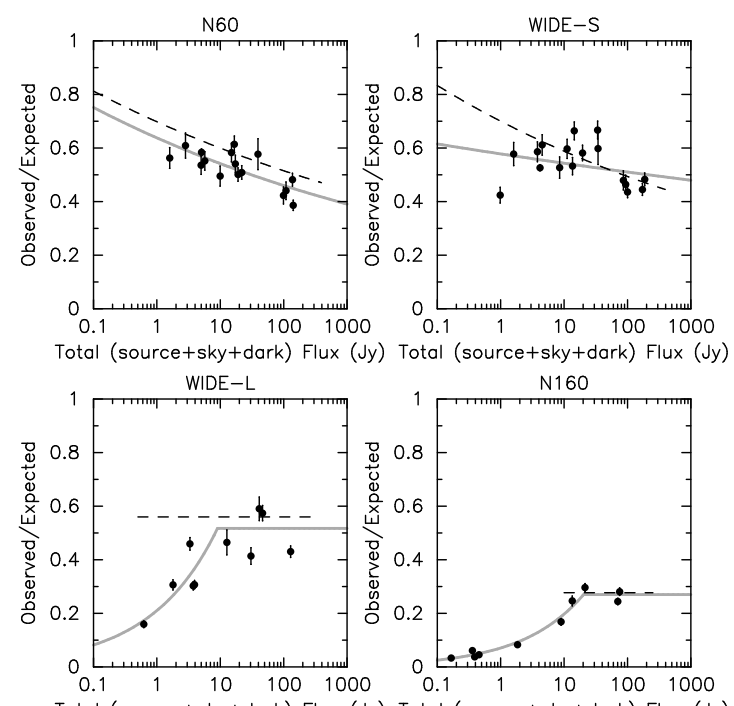

Figure 1: The observed over expected flux ratio vs. the Total (Observed plus sky and dark) flux plot. The dashed lines represent the fit determined by assuming a point source (i.e., using an aperture correction factor) $[14]$ and the gray solid lines are the fits determined by using a $3 \sigma$ contour aperture [15] in obtaining the total observed flux.

([13], and references therein). This response effect was quantified for point sources [14]. For point-source flux calibration, source fluxes are measured only from the PSF core of the photometric calibration sources (solar system objects and stars) and the flux from the PSF wing is recovered by using an aperture correction scaling factor based on the theoretical PSF shape. Then, the slow transient response correction was determined by comparing the ratio between the observed and expected total fluxes. This correction method, by design, works only on point sources because it assumes a specific surface brightness profile (i.e., a Gaussian PSF).

For extended sources, a specific surface brightness profile cannot be assumed a priori. Hence, we measured the observed total fluxes of point-source reference targets by using a contour aperture at the $3 \sigma$ surface brightness level, and compared them with their expected total fluxes. Figure 1 shows the slow transient response (the ratio of the observed to expected total fluxes) as a function of the total flux for both point sources (dashed) and extended emission objects (solid) [15]. For the present compilation of FIS slow-scan mapping data for extended sources, we used the extended source method to calibrate our flux measurements.

\section{Color correction and SED fitting}

The FIS fluxes are then fit with a modified blackbody, $F_{V} \propto \lambda^{-\beta} B_{V}(T)$, to determine the color correction factor to derive the completely-corrected observed fluxes of the target sources. Together with photometry at different wavebands covering most of the spectral energy distribution (SED), it is now possible to characterize the thermal emission properties of these target sources. Figure 2 presents an example of such a fit for U Hydrae, which is a well-studied AGB star ([16] and references therein). Previously a dust temperature of $45 \mathrm{~K}$ was reported. Our fitting suggests a slightly colder dust temperature of $42 \mathrm{~K}$ with a larger $\beta$ of 1.94 . This reduced dust temperature is a result due to the updated flux values especially at the longer wavebands based on the following: (1) using optimally reduced maps with less noise, a larger amount of colder dust was detected, and (2) updated slow transient response functions optimized for extended emission yielded better flux measurements for longer wavebands. 


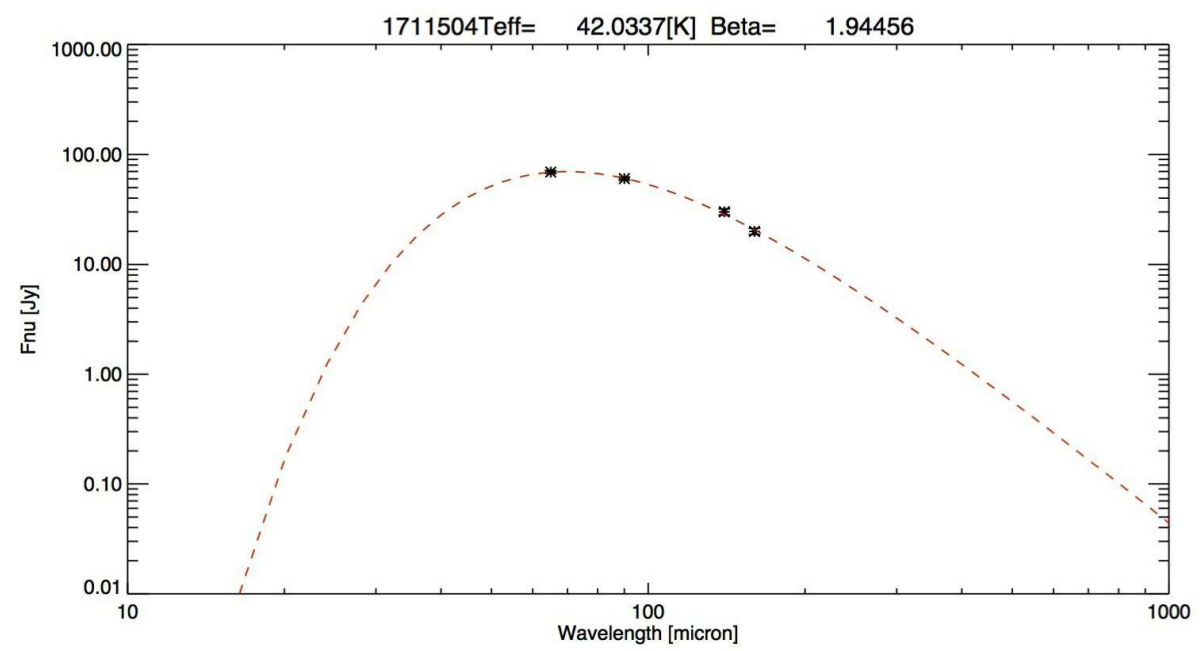

Figure 2: The SED fit for U Hydrae (the error bars are smaller than the symbols) resulting in an effective dust temperature of $42 \mathrm{~K}$.

\section{References}

[1] G. Neugebauer, et al., The Infrared Astronomical Satellite Mission, ApJ Letters, 278, L27 (1984)

[2] K. Young, et al., Circumstellar Shells Resolved in IRAS Survey Data. II, ApJ, 409, 725 (1993)

[3] M. F. Kessler, et al., The Infrared Space Observatory (ISO) mission, A\&A, 315, L27 (1996)

[4] I. Yamamura and T. de Jong, Warm Gas Around AGB Stars, ESA Special Publication, 456, 155 (2000)

[5] H. Izumiura, et al., A Detached Dust Shell Surrounding the J-type Carbon Star Y Canum Venaticorum, A\&A, 315, L221 (1996)

[6] T. Ueta, et al., A Hubble Space Telescope Snapshot Survey of Proto-Planetary Nebula Candidates: Two Types of Axisymmetric Reflection Nebulosities, ApJ, 528, 861 (2000)

[7] M. W. Werner, et al., The Spitzer Space Telescope Mission, ApJ Supplement Series, 154, 1 (2004)

[8] H. Murakami, et al., The Infrared Astronomical Mission AKARI, PASJ, 59, 369 (2007)

[9] G. L. Pilbratt, et al., Herschel Space Observatory. An ESA Facility for Far-Infrared and Submillimetre Astronomy, A\&A, 518, 1 (2010)

[10] M. Kawada, et al., The Far-Infrared Surveyor (FIS) for AKARI, PASJ, 59. 389 (2007)

[11] N. Ikeda, FAST User's Handbook, ver. 0.1, ISAS/JAXA (2012)

[12] E. Verdugo, et al., AKARI FIS Data User Manual, ver. 1.3, ISAS/JAXA (2007)

[13] H. Kaneda, et al., Transient response of stressed Ge:Ga detector for ASTRO-F, Advances in Space Research, 30, 2105 (2002)

[14] M. Shirahata, et al., Calibration and Performance of the AKARI Far-Infrared Surveyor (FIS) Slow-Scan Observation Mode for Point-Sources, PASJ, 61, 737 (2009)

[15] R. L. Tomasino, et al., Calibration of the AKARI FIS for Extended Emission, in SPICA Science Conference 2013, in press

[16] H. Izumiura, et al., Extended dust shell of the carbon star U Hydrae observed with AKARI, A\&A, 528, A29 (2011) 